\title{
Aggregate Collaborative Planning in Non-hierarchical Business Networks
}

\author{
Ricardo Almeida, César Toscano, Luis Carneiro, and Américo Azevedo \\ INESC Porto, Rua Dr. Roberto Frias, 378, 4200 - 465 Porto, Portugal \\ ralmeidalinescporto.pt
}

\begin{abstract}
The last decade has been characterized by times of change, including increasing levels of globalization and competition. Collaboration allowed SMEs to respond effectively to the development of new complex products and services but presented new barriers concerning the management of their business processes and relations with partners. This paper presents a new collaborative aggregate planning for non-hierarchical business networks, where SMEs collaborate in the selection of partners and definition of the operations plan required to specify, manufacture and distribute a custom made products. The proposed approach is based on negotiations undertaken by the partners in order to promote the creation of alternative operations plans and allow a multicriteria evaluation of the involved costs and delivery dates. The proposed approach promotes the reduction of communication between partners, a reactive analysis of changes to planning and associated impact, enhancement of trust (since all partners have the same weight on the decision process).
\end{abstract}

Keywords: Collaborative, virtual organization, aggregate, planning.

\section{Introduction}

Manufacturing companies are facing one of the most difficult periods after Industrial Revolution. The worldwide crisis has reduced significantly the levels of market consumption, having a high impact on the resellers' orders increasing even more competition levels. On the other side, market demand for innovative and complex products and services, require more agile organizations, leading to unique opportunities for SMEs.

This new business reality required a higher flexibility for companies and forced them to focus on business processes integration (internal and external to their organization), aiming to reduce the effort on virtual organization's management (like setup and delivery times, changes' control and associated impact evaluation to the planning, etc). Companies searched for methods and tools which could help on the support of sustainable decisions and become more reactive to market uncertainty.

This research work addresses particularly the collaborative aggregate planning of operations that takes place during the formation of non-hierarchical business networks, supporting the definition of lead times and costs for custom made and complex products. 
This work presents (on the first two chapters) the scope and areas of research, which resumes the methodology applied and definition of concepts related with collaborative networks. The next chapters focus on the possible approaches for collaborative planning and the proposed solution to deal with aggregate planning (which derives from a high-level product design phase). Finally, this work ends with the major conclusions and future works.

\section{Background}

This research was developed under the European RTD project Net-Challenge [Ref.: FP7-CP-FP229278-2], whose goal is to design and develop an integrated framework to support SMEs implement new business models and establish dynamic and nonhierarchical business networks for complex products manufacturing.

The scope definition and requirements for this research work were extracted from the analysis of the six companies involved in the project; two belonging to the textile and garment sector, two from the shoe industry and the last two from the machine tool industry.

Some authors [3] resumed these requirements and proposed, as methodology for non-hierarchical collaboration networks, two main levels to be considered: management of the Business Community activities and management of the temporary Virtual Organization. Regarding answering to these two levels, the author presented four major phases: Build, Form, Operate and Dissolve. The first and last phases to respond to the first level; and the second and third phases to respond to the last level.

During the Form phase, partners collaborate to specify the product, find solution for the customer needs and set up a price and lead-time to manufacture the production batch. For this research work, the focus has been dedicated to this phase, concerning to collaborative planning and partner's quotation evaluation, which requires ICT tools to support managers in decision making.

\section{Related Research Areas}

A collaborative network $(\mathrm{CN})$ is constituted by a variety of entities (organizations and people) that are largely autonomous, geographically distributed and heterogeneous in terms of their operating environment, culture, social capital and goals [1]. Usage of computer networks to support collaborative networks and the shared belief of a winwin relationship to achieve a goal which would never be possible working individually are some of their major characteristics. When integrating such kind of networks, companies aim to build a new organization of larger dimension, access new markets and produce new products and services, share risks, develop synergies for investigation and supply a wider service (upstream and downstream) for their customers. These kinds of networks are characterized by an intensive exchange of detailed data with its suppliers and customers who may consider, for example, actual and future load of machines, availability of resources, forecasting and orders' progress. 
Considering governance model, business networks can be hierarchical or nonhierarchical [4]. The hierarchical relationship (hierarchical network) is characterized by the existence of one leading partner that controls the network and settles the operational rules. It is the superior partner that makes the rules and dictates. In a nonhierarchical network, all partners have the same status, that is to say, no one has a special position or leads the network. Therefore, all decisions affecting the partnership are mutually agreed upon. In this type of networks, one partner may assume the coordination responsibilities, but has no dominant status over the other partners.

\section{Collaborative Planning Approaches}

Production planning is one of the most critical business processes on traditional industrial companies and assumes the same importance on CNs. The major challenge of production planning on $\mathrm{CNs}$ arises of how to link and coordinate planning between the different parts that comprise the network. The need for a process of negotiation (coordination scheme between two or more parties) was detected by several authors, to ensure the synchronization between production plans from distinct organizations.

\section{Planning as a negotiation process}

Some authors defined a coordination scheme, through which plans of two or more decision-making units are aligned [5]. This scheme requires all members to adapt their individual plans in order to be developed an overall planning acceptable by all supply chain members, and refusing to follow any centralized planning approach that do not consider an active contribution of all partners. The same author defined Collaborative Planning (CP) as a joint decision making process of individual ("local") production plans (supply chain partners), with the aim of achieving coordination to create an overall optimized solution for a business opportunity. CP might also be defined as a connection of planning processes; local to their planning domains, but that collaborates in order to create a common and mutually agreed upon plan [7]. This collaboration is achieved through an exchange of relevant information. Thus, input data is updated faster and planning results become more accurate.

Collaborative planning involves activities by means of which individuals coordinate their planning processes [6]. Individuals predominantly plan according to the goals of their own department, but in cooperative relationships they additionally try to create possibilities for the optimization of others' planning. Thus, CP includes aspects that enable the other person to recognize how individual plans should be adapted, according to which criteria the planning can be optimized, and which restrictions in the common planning must be accepted. In short, $\mathrm{CP}$ describes how individuals are able to orient their plans towards each other to reach a joint optimization of the planning across departmental boundaries.

\section{Centralized and decentralized planning approaches}

The choice of applying a collaborative approach has revealed several challenges to organizations. One of the most challenging is the idea of a centralized planning that must be followed and achieved to guarantee the overall success. Some authors presented some doubts about the appropriateness of a centralized planning approach [8]. Some of the constraints pointed by these authors are the multi-network 
involvement of partners (since they participate on several networks, the probability of interference on their planning activities is extremely high) and unwillingness to share information (most of the time, companies are not available to share information about resources and capabilities).

When working on a centralized planning approach, all decisions lead to an optimal solution at a first try, since the calculation is provided by a unique entity, based on the theoretical capacity of each partner. A lack of transparency of the overall decisionsituation leads to poor coordination in decisions involving several of these intraorganizational planning domains [8]. To establish coordination in this situation, centralized collaborative planning solutions provide remedy by offering decision models and processes. The delivery time calculation is performed using traditional sequence algorithms, assuming each partner as a local resource that is fully available. Such kind of planning approaches are only possible when the supply chain partners belong to the same group of companies or when partners have a dedicated capacity (which is assumed from the beginning of the business opportunity). This approach was defined as hierarchical organized coordination [9].

When working with actual and complex business scenarios (like the textile sector, for instance), the centralized approach presents several constraints, like the loss of local autonomy, which is not accepted by most companies. Supply chains are most commonly planned and controlled on a decentralized basis, which are less advantageous and profitable than the centralized approach [8]. The coordination required for this planning approach is provided via bilateral negotiations with relevant partners on the supply chain [9]. The authors assume that the first step should be the definition of the best planning for the organization; then, it should only depend on the direct customers and direct suppliers. This could reduce drastically the conflicts and the loss of confidential information.

\section{Aggregate Collaborative Planning}

\subsection{Scope and Major Requirements}

Business companies have revealed that a daily major concern is dedicated to manage the articulation between their local productions planning with the requirements of their customers, especially when depending on partners' responses about their own availability [2]. According to key-users of these companies, this is one of the most expensive processes due to the high number of activities dedicated for control and communication (considered as non-value added activities) as well the required number of iterations to achieve a common agreement (between the internal production plan and partners' availability).

Another important issue presented by these key-users [2] was the need of a quick and accurate response to customer's requirements about delivery date and cost, which occur in two different moments. The first moment is triggered after the contact of the customer with one company, where the business opportunity is presented and a first prediction on delivery dates and costs is requested from the company. Usually, the company analyses the business opportunity and (when doesn't have enough knowledge about all manufacturing phases) requests some help to other companies to define the major product design phases and to calculate the needed time for each 
operation. The next step includes the definition of precedence between all operations and, finally, a rough plan is developed according to the theoretical capacities of each company (defined at the beginning of each year), to calculate the delivery time. Regarding prices, the cost of all operations are summarized. Finally, the proposal is sent to the final customer.

The second moment is triggered when the company receives the customer order. The business case company requests the participation of the other companies (contacted on the first moment) in order to validate if the previous agreements (considering cost and availability) remain valid for the customer order. A detailed approach is performed, taking the product design phase to lower levels of the product structure, defining detailed bill-of-materials and routings. A high number of technical documents are exchanged requiring many activities of control and communication. Also the availability of each partner is validated in this moment, asking for each partner to confirm their capacity and propose new dates if necessary. Every change (to the defined plan) needs to be evaluated (since each company analysis the new proposal on their local planning) requiring a great effort for to achieve a common understanding. Also, since this planning is not visible to all companies, it's very difficult to work for an optimal global solution, increasing the number of iterations to achieve a final agreement. The same situation occurs anytime a change is requested by one partner, for example, due to a delay of his raw material supplier. In this case, the partner will not follow the predicted plan, and all members need to re-adjust their plans.

The choice of the best alternative plans was also referred by key-users as a difficult activity, due to several aspects. One of the presented arguments was related to the criteria used (and the associated indicators) which differ from the type of business, the company's goals and even the type of business opportunity. Besides this complex approach, it was also referred the different types of data assumed for each indicator and the high number of possible plans (due to the combinations of different partners for each operation) which make almost impossible, for key-users, to select the best proposal without using empirical techniques. Key-users are aware of the uncertainty of empirical decisions and desire an effective method to help them on this type of decisions.

\subsection{Multi-criteria Planning Methodology}

Net-Challenge's project designed a collaborative aggregate planning based on a negotiation process, as a response to the needs of the first phase of the production planning (described on the previous chapter). The term "aggregate" was used to express the definition and planning of major operations (and not detailed operations planning for shop floor control) which are assigned to partners in the virtual organization (VO) being formed, leaving the operation's details for partner's own management. This approach is based on a decentralized negotiation model, which allows partners to propose new delivery dates and costs, represented graphically at Image 1.

The proposed approach for aggregate collaborative planning includes two steps:

- The customer presents the business opportunity requirements to the Broker (company that identified the business opportunity and is responsible for the interaction with the customer). After the elicitation of the major requirements 
on the required custom made product, the product concept/design is defined by the Broker and by the new partners that are invited to join the VO, based on their specific competencies and availability [3]. These partners are called "core partners" since they integrate Product Design activities and share some risk in responding to the overall business opportunity. In this context, the necessary main operations of the virtual organization are defined by the Broker and core partners, with its sequence and timing restrictions (start, finish and leading times), taking into account the due date specified or expected by the customer and theoretical capacities defined on each partner's profile.

- In the beginning of the planning process the criteria for partner selection are defined and agreed by core partners.

- Additional partners (called "potential partners") are invited to participate on several operations. Their selection is performed according a criteria (for example, certification or feedback from previous negotiations) previously defined by the Broker and core partners. This gives rise to a first operations "rough" plan that the Broker proposes to all the members in the VO;

- Partners receive the first "rough" plan and a set of requests for quotations, one for each operation allocated to him. Each partner proceeds then to a local analysis of their local production capacity to evaluate if it is possible to accept the proposed dates and lead time for the indicated quantity. A quotation is then formulated, accepting the proposed plan or proposing an alternative for the request.

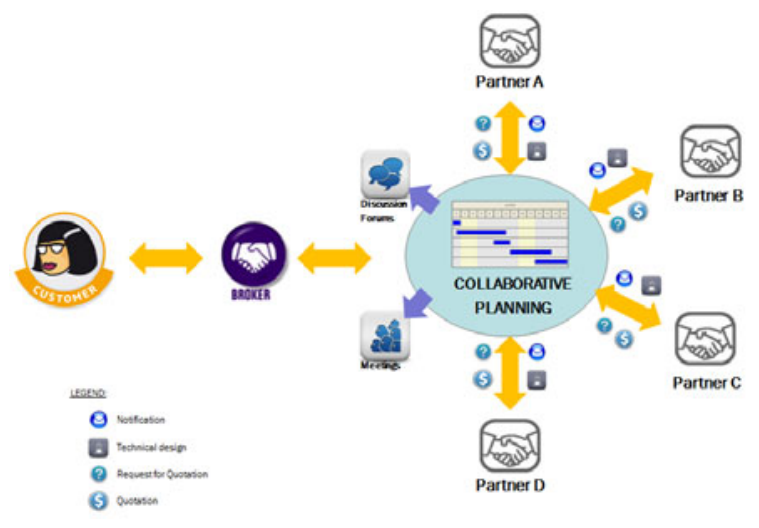

Fig. 1. Aggregate planning approach

This approach, although centralized when considering the supporting ICT architecture (for the graphical view and management of requests and quotations), promotes a decentralized approach on the definition of the VO's planning, since each partner is capable of proposing and participating actively on the characterization of each required operation and to request from any other partner a change on the plan (through the creation of a request for quotation).

The major characteristic is the inexistence of a major partner imposing a plan to the others; every core partner has the same "weight" on the decision process over the 
operations planning of the network (major condition for non-hierarchical networks). The Broker has the responsibility to coordinate this process and promote the resolution of any conflicts. Major advantages can be resumed as follows: reduction of the "traditional" communication, like e-mails and phone calls, between partners (since the planning is shared and available for any partner in the VO); accurate control of any change and evaluation of the associated impact, with the ability to apply the necessary events to handle those changes; enhancement of trust, due to the increased visibility and to the fact that core partners have the same weight on the decision process. Each change proposed by any member in the VO will create new possible plan which, after been analyzed and accepted by the involved partners, will create a new (accepted) plan which will be evaluated following common criteria (defined in the very beginning of the process); this topic is explained on the next chapter.

\subsection{Solutions Quality Evaluation}

Each partner can propose new delivery dates, lead times and costs, directly on a webbased planning graphical tool which is available and shared by all VO's partners. Every time a partner proposes a change on a given operation, he is (in fact) asking the affected partner to accept this change (and declare its cost) or to make a counterproposal. Each negotiation round correspond to a pre-defined time period (for example, 2 days) available to discuss/negotiate delivery times and costs, allowing partners to present quotations for each request-for-quotation (RFQ) performed by other partners. For each change proposed (which "triggers" RFQs to all involved partners), it is asked to the partners to present quotations which might answer, integrally, to the asked RFQ or even suggest new changes. When a proposal has $100 \%$ agreement of all partners ("no pending notifications") it will be considered a plan; although it still allows to be changed by any VO's partner, as long as the negotiation time period doesn't expired. This plan is serialized according multicriteria (previously defined by the Broker and core partners). If the negotiation time period expires and some changes are still waiting for partner's quotations, they will be avoided and it will only be invoked the "effective" plans. Image 2 presents a flowchart representing the steps taken to create a plan. An overall scheme is presented on Image 3, representing a negotiation round.

The criteria to be used are, collaboratively, defined by the Broker and core partners in the initial setup of the VO, thus specifying the criteria that will support subsequent decisions in the process. For each of these criteria, it's possible to define a degree of importance, using a percentage score as a final weighting factor, which will used for calculate the best partner proposal. So each criteria considered do not have the same importance but each has an importance expressed by a ranking system. One of the major concerns of the criteria definition is to include criterions which maximize the probability of gain of the customer's proposal and not only the internal efficiency of the network. This is an important step to assure the requirements of nonhierarchical networks.

The requirements analysis carried out with business case companies confirmed the two criteria are considered is most situations: price and delivery date. However, in the model proposed, it is included additional criteria to promote a wider approach and business application. 


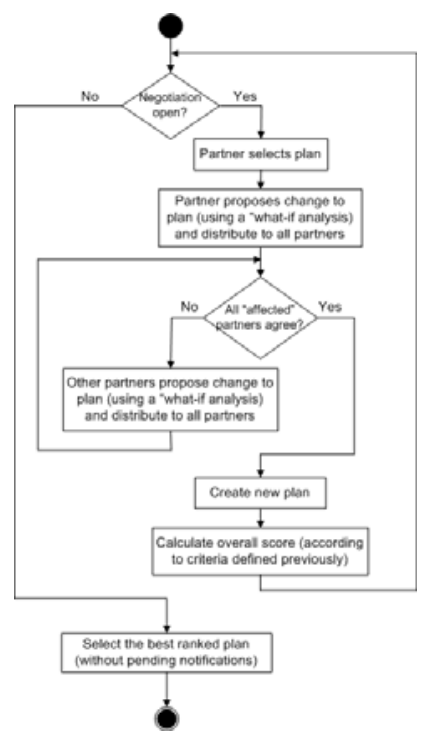

Fig. 2. Creation of an alternative plan

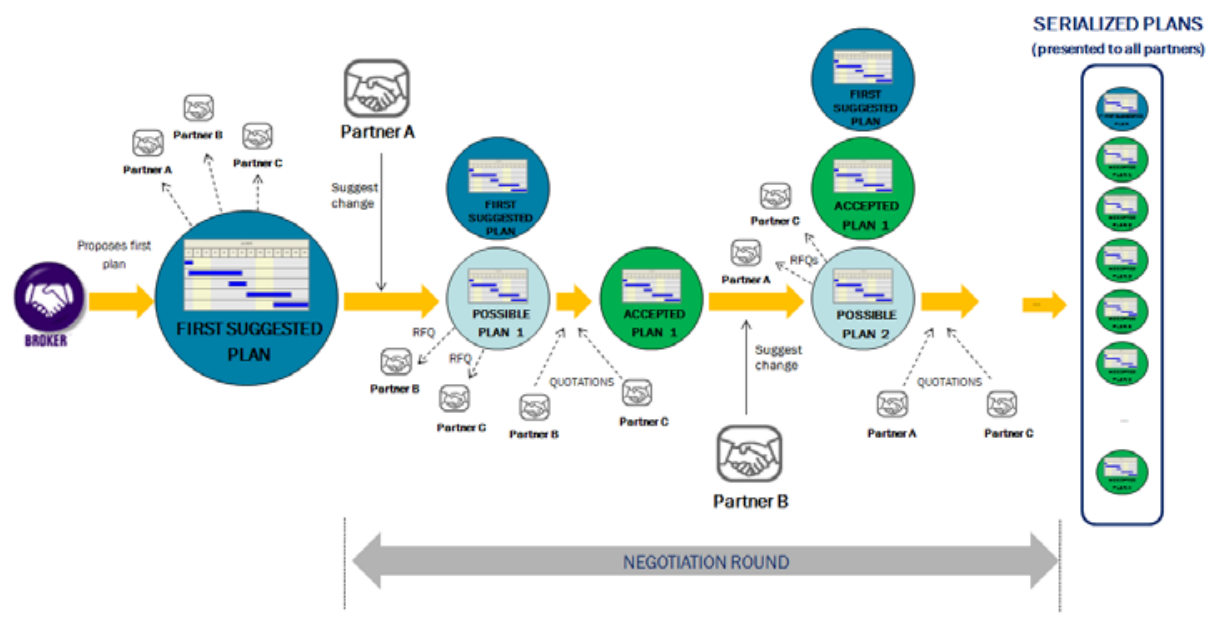

Fig. 3. Negotiation round

In order to perform a multi-criteria analysis and assist in decision making, normalization criterion must be defined and used. For the case in question, linear normalization was selected. The basic principle for this standardization was the definition of minimum and maximum values, and assignment of simple formulas for the normalization of attributes for benefits and costs. For each indicator, it must be defined if it's considered a cost or benefit, in order to apply different formulas (presented on Image 4). 


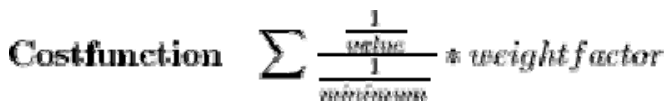

Benefitfunction $\sum \frac{\text { valwe }}{\text { maximum }}$ weight foctor

Fig. 4. Formulas used for attributes' normalization

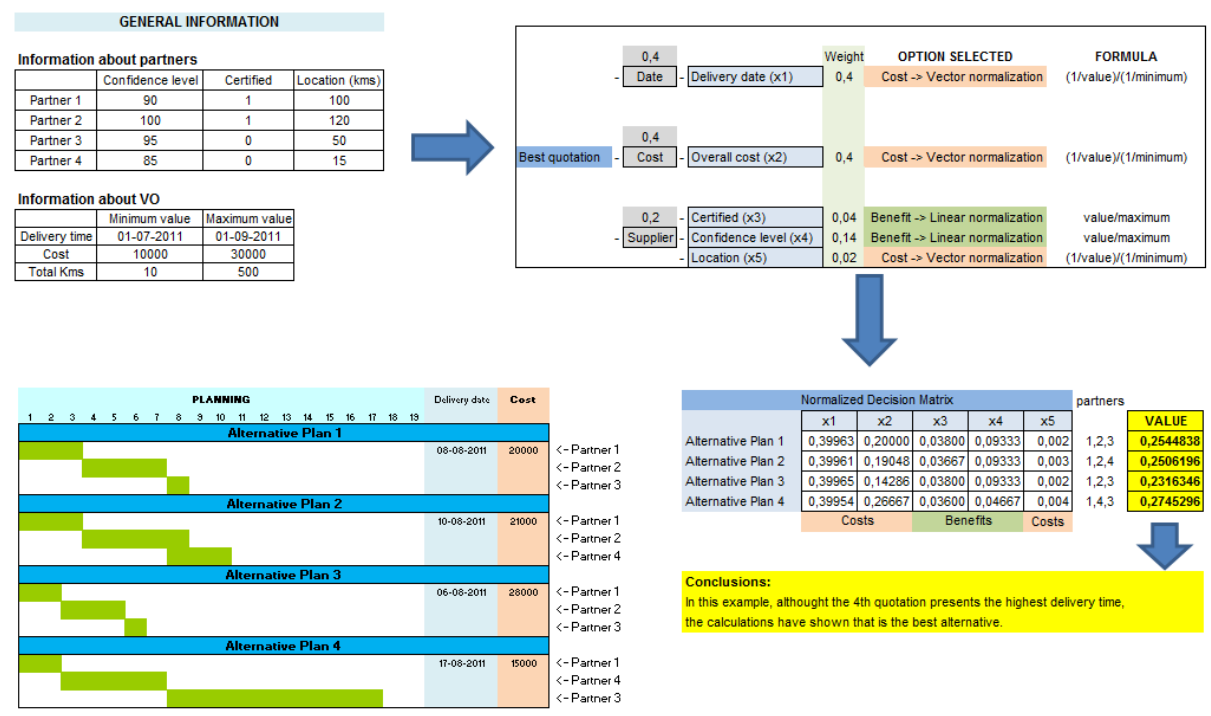

Fig. 5. Example of algorithm calculation for evaluation of alternative plans

All values are summed (costs and benefits), resulting in a value between 0 and 1 , which represents the score of the alternative plan (closest to 1 , the better result).

Image 5 shows an evaluation of four alternative plans, considering five criteria elements: delivery date, cost, confidence level, partner's certification and location. The tables presented on the left side of Image 5 present the indicators and the Gantt charts of the four possible plans being evaluated. The tables on the right side of the same image resume the formulas used (for benefit and costs) and the calculation for the normalization process. The algorithm is responsible for data normalization (applying the related formulas) and applies the weight factor for the indicators of each alternative plan. As final operation, the algorithm sorts the results, defining the best plan to the higher result retrieved (closest to 1).

\section{Conclusions and Further Developments}

In this research work, it has been studied several concepts related with virtual business networks and collaborative planning processes. Integrated on the NetChallenge European project it proposes an innovative collaborative planning approach, based on a truthful concept of negotiation between partners. It includes the definition and organization of operations and a concrete set of activities, with the goal 
of defining and organizing the operations that are required by the product's high level design, and given the customers' specific requirements (delivery date and price among others). The proposed approach reveals several major advantages, like the reduction of the traditional communication means (since all information is centralized and shared by all partners), ability of partners to suggest new delivery dates in a decentralized negotiation model, evaluations of alternative plans based on multicriteria and a guarantee of partner's data privacy, since other partners don't access to partner's local planning information. This approach assumes a major role for managers to provide sustainable decisions on their companies. At the moment, the presented approach is being developed by Net-Challenge's partners and will be tested on the business case companies described.

As future work, it's expected to validate the proposed approaches on several industries and evaluate the possibility of including new capabilities of integration with local information systems (in order to allow managers for an automatic production capacity validation with their local production planning).

Acknowledgements. The authors would like to acknowledge the co-funding of the European Commission within NMP priority of the Seventh RTD Framework Programme for the Net Challenge project (Innovative Networks of SMEs for Complex Products Manufacturing). The authors also acknowledge the valuable collaboration provided by the project team during the research work.

\section{References}

[1] Camarinha-Matos, L.M., Abreu, A.: A contribution to understand collaboration benefits. In: Emerging Solutions for Future Manufacturing Systems. IFIP, vol. 159, pp. 281-298 (2005)

[2] Carneiro, L., et al.: An innovative framework supporting SME networks for complex product manufacturing. In: Collaborative Networks for a Sustainable World, vol. 336, pp. 204-211 (2010), doi:10.1007/978-3-642-15961-9_24

[3] Kankaanpää, T., Shamsuzzoha, A., Carneiro, L., Almeida, R., Helo, P., Fornasiero, R., Ferreira, P.S., Chiodi, A.: Methodology for non-hierarchical collaboration networks for complex products manufacturing. In: Collaborative Networks for a Sustainable World. IFIP AICT, vol. 336, pp. 204-211 (2010), doi:10.1007/978-3-642-15961-9_24

[4] Pisano, G.P., Verganti, R.: Which kind of collaboration is right for you? Harvard Business Review (USA) 86(12), 78-86 (2008)

[5] Stadler, A.: A framework for collaborative planning and state-of-the-art. Springer, Heidelberg (2007)

[6] Windischer, A., et al.: Characteristics and organizational constraints of collaborative planning. Springer, Heidelberg (2007)

[7] Kilger, C., Reuter, B.: Collaborative Planning. In: Supply Chain Management and Advanced Planning, Concepts, Models, Software and Case Studies, pp. 259-278 (2005)

[8] Breiter, A., et al.: Coordinarion in Supply Chain Management - Review and Identification of directions for future research. In: Logistik Management, Systeme, Methoden, Integration, pp. 11-42. Springer, Heidelberg (2009)

[9] Fischer, J.-G., Gneiting, P.: Collaborative Planning Processes. In: Build to Order - The Road to the 5 Day Car. Springer (2008) 